\title{
Arima Forecasting Model for Uganda's Consumer Price Index
}

\author{
Yeko Mwanga \\ School of Statistics and Planning, College of Business and Management Sciences, Makerere University, Kampala, Uganda
}

\section{Email address:}

yekomwanga@gmail.com

\section{To cite this article:}

Yeko Mwanga. Arima Forecasting Model for Uganda's Consumer Price Index. American Journal of Theoretical and Applied Statistics. Vol. 9, No. 5, 2020, pp. 238-244. doi: 10.11648/j.ajtas.20200905.17

Received: September 1, 2020; Accepted: September 23, 2020; Published: October 12, 2020

\begin{abstract}
In Uganda, the Central Bank watches closely inflation which happens to be one of the key macroeconomic indicators for which the central bank rate is anchored on. Uganda Bureau of Statistics disseminates monthly Consumer Price Indices (CPIs) to the various stakeholders. Currently, the CPI is computed for eight urban centres spread across the country. The monthly CPIs serve mostly those users who require past and current inflation rates. The main objective of this study is to identify and estimate an ARIMA model for the CPI and use it to make short term forecasts. We relied upon monthly Consumer Price Indices from January 2010 to July 2020 obtained from Uganda Bureau of Statistics. The time series was transformed so as to make it stationary, before identification and estimation of ARIMA (p, d, q) x (P, D, Q) $)_{12}$ models. An ARIMA $(1,1,1)(0$, $1,1)_{12}$ with no constant was selected as the best model, because it had the least AIC and BIC. Additionally, all the coefficients of the ARs and MAs were significant at $1 \%$ level. Using the selected model, inflation forecasts were generated for 12 months (August 2020 to July 2021) and found to fluctuate between 4.7 and 6 percent. We recommend this model to Uganda Bureau of Statistics and Central Bank to use it to make forecasts and disseminate them to users. In conclusion, generally good forecasts are vital for better resource allocation, planning and decision making.
\end{abstract}

Keywords: Consumer Price Index, Inflation, ARIMA Model, Forecasts, Uganda

\section{Introduction}

The monetary and fiscal authorities in any country closely monitor key macroeconomic indicators. These indicators comprise of economic growth, unemployment, balance of trade and inflation. Knowledge of such indicators is the basis for making informed decisions and sound judgment regarding investment and allocation of scare resources [1]. In Uganda, the Bank of Uganda is the apex bank which regulates and supervises commercial banks and other non-bank financial institutions. The Bank of Uganda adjusts the central bank rate $(\mathrm{CBR})$ as a policy tool in line with inflation rates with the aim of influencing commercial banks and other money lenders to adjust their interest rates [2, 3]. The Bank of Uganda issues monetary policy statements monthly soon after Uganda Bureau of Statistics (UBOS) releases the previous month's consumer price index (CPI). Currently, the CPI covers eight urban centres spread across the country. These centres are: Kampala, Jinja, Mbale, Masaka, Mbarara, Fort Portal, Gulu and Arua [4].

The policy makers, planners, investors and other stakeholders are interested in overall inflation because it throws some light on price movements in the national economy. Inflation is also useful for: wage indexing and negotiations, deflating the gross domestic product (GDP), costing of contracts, investment decisions among others [5]. UBOS monitors the headline inflation and its sub-components of food, core and energy, fuels $\&$ utilities. Food inflation is mainly driven by weather patterns (first rainy season runs from February to June/July and the second from August to December) experienced annually in most parts of the country [6].

The current monthly CPIs serve mostly those users who require past and current inflation rates. Investors, traders and other economic actors make decisions based upon both current and forecasts of CPI. No inflation forecasts are usually released during the monthly dissemination of the CPI in Uganda. Making good predictions is not easy and as such a number of researchers have come up with different models and analytical techniques which may be applied to generate reliable forecasts [7, 8]. Montgomery et. al applied the Box Jenkins Methodology using Autoregressive Integrated Moving 
Average (ARIMA) model to make business forecasts [10].

This study attempted to come up with forecasting models and short run forecasts for headline inflation. The main aim of the study was to estimate an ARIMA forecasting model for inflation. More specifically, the study identified the most appropriate forecasting model, then used it to generate a few monthly forecasts.

\section{Literature Review}

The governments worldwide are faced with a number of challenges some of which are unprecedented in nature like corona viruses pandemics ravaging the world and credit crunches among others. These factors have generally contributed to economic slowdowns, loss of employment and worsening of balance of payments especially for developing countries. In order promote economic growth the governments including Uganda promote foreign direct investment through a number of incentives such as those spelt out in the Investment Code Act 1991 [1, 2]. Furthermore, the macroeconomic environment had to be stable which includes control of inflation.

Time Series forecasting is a feature in a number of fields, including: business, government statistics, economics, medicine and social science [9]. Time series forecasting models are basically mathematical techniques which rely upon historical series to make forecasts. Forecasting may be classified into short term, medium term and long term depending on the period in focus. Short-term forecasting problems involve predicting events only a few periods (days, weeks, and months) into the future whereas medium-term forecasts extend for 1 to 2 years into the future. The longterm forecasting problems can may extend for a number of years. UBOS makes monthly releases of the Consumer price index with virtually no forecasts [4].

A number of researchers have used Autoregressive Moving Average (ARIMA) Models on Consumer Price Index series to make forecasts for developing and developed countries. Habimana et. al estimated an $\operatorname{ARIMA}(4,1,6)$ model for Rwanda which was used to make monthly forecasts for January to December 2016 [12]. Similarly, Adams et. al came up with ARIMA $(1,2,1)$ to make CPI forecasts for Nigeria [14]. On the basis of the German CPI time series 1960 to 2017, Nyoni modeled using ARIMA and made forecasts for seven years [15]. Likewise, Zhang used ARIMA model on China CPI series for the period January 2008 to December 2016 to make forecasts [13]. Apparently, the literature point to unique ARIMA forecasting models for every country which implies that no one model fits all countries. It is therefore, inevitable for us to estimate an ARIMA forecasting model for the Uganda CPI series.

\section{Materials and Research Methodology}

This study relied on the monthly Headline Consumer Price Index series from January 2010 to July 2020 obtained from Uganda Bureau of Statistics (UBOS). The reference period for this series was $2009 / 10=100$. An Autoregressive Integrated Moving Average (ARIMA) method was used to model the Consumer Price Index data [7]. This model relied upon differencing to transform the non-stationary series to a stationary one and autocorrelation functions (ACFs) and partial autocorrelation functions (PACFs) for identification of the ARIMA model. The ARIMA modeling procedure has four major steps, namely: identification, estimation, diagnostic checking and forecasting.

\subsection{Identification}

A time series is said to be stationary if it has a constant mean and variance. A series is non-stationary if it has to be differenced and at times it may have to be transformed more than once in order to realize stationarity $[7,9]$. The headline CPI series was made stationary by first differencing, then a possible Autoregressive Moving Average (ARIMA) model was identified. The process of identification started with the generation of autocorrelation functions (ACFs) and partial autocorrelation functions (PACFs). The ACFs and PACFs for the time series are computed as follows:

The autoregressive model of order $p, A R(p)$ is as follows:

$$
y_{t}=\alpha_{0}+\alpha_{1} y_{t-1}+\alpha_{2} y_{t-2}+\cdots+\alpha_{p} y_{t-p}+\varepsilon_{t}(1)
$$

Where:

$y_{t}=$ actual series at time $\mathrm{t}$

$\alpha_{i}=$ autocorrelation parameter $y_{t-i}$

$\varepsilon_{t}=$ irregular term at time $\mathrm{t}$

And the moving average model of order q, MA (q);

$$
y_{t}=\beta_{0}+\varepsilon_{t}-\beta_{1} \varepsilon_{t-1}-\beta_{2} \varepsilon_{t-2}-\cdots-\beta_{q} \varepsilon_{t-q}
$$

Where:

$y_{t}=$ actual series at time $\mathrm{t}$

$\beta_{i}=$ autocorrelation parameter $\varepsilon_{t-i}$

$\varepsilon_{t}=$ irregular term at time $\mathrm{t}$

The autoregressive and moving average model of order $p$ and $\mathrm{q}, \operatorname{ARMA}(\mathrm{p}, \mathrm{q})$ is a combination of the AR (p) and MA $(\mathrm{q})$, and stated as:

$$
\begin{gathered}
y_{t}=\alpha_{1} y_{t-1}+\alpha_{2} y_{t-2}+\cdots+\alpha_{p} y_{t-p}+\beta_{0}+\varepsilon_{t}-\beta_{1} \varepsilon_{t-1}- \\
\beta_{2} \varepsilon_{t-2}-\cdots-\beta_{q} \varepsilon_{t-q}
\end{gathered}
$$

Where:

$y_{t}=$ actual series at time $\mathrm{t}$

$\alpha_{i}=$ autocorrelation parameter $y_{t-i}$

$\beta_{i}=$ autocorrelation parameter $\varepsilon_{t-i}$

$\varepsilon_{t}=$ irregular term at time $\mathrm{t}$

The series was found non-stationary and as such it was first differenced to achieve stationarity, thereafter the series had to be integrated. Therefore $d$ was introduced in the model, the order of integration and the model became an Autoregressive Integrated Moving Average (ARIMA) expressed as ARIMA (p, d, q). Since the series was monthly, it introduced a possibility of seasonality and the model therefore may be expressed as $\operatorname{ARIMA~(p,~d,~q)~(P,~D,~Q).~}$ The $P$ represented the number of parameters of the seasonal AR component while $\mathrm{Q}$ represented the parameters of the 
seasonal MA and D the number of differencing of the seasonal component to realize stationarity.

The ACFs and PACFs were used to determine if the series was stationary. After differencing to obtain stationarity, the ACFs and PACFs were used to determine the type of model to be estimated and the number of parameters to be included in the model.

\subsection{Estimation}

A nonlinear iterative process was used in the estimation of parameters. The possible models were identified and estimated. The model with the least Akaike Info Criterion (AIC) and Schwarz Bayesian Information Criterion (BIC) values was selected. The t-statistic was used to determine the significant coefficients.

\subsection{Diagnostic Checking}

The fitted models were checked for appropriateness by testing the residuals for normality using the Bartlett's test for white noise [7, 9]. Once the residuals were normally distributed with zero mean and constant variance then the model was considered appropriate for estimation and it was therefore selected. The histogram of the residuals of the selected model were also computed.

\section{Results}

\subsection{Stationarity Tests}

Stationarity of the time series is required before fitting an ARIMA model. It is evident from Figure 1, that the Headline Consumer Price Index was trended. However, in order to confirm if the series was indeed trended/non-stationary a unit root test was performed. The hypothesis tested was that the series had a unit root against the alternative that the series does not have a unit root.

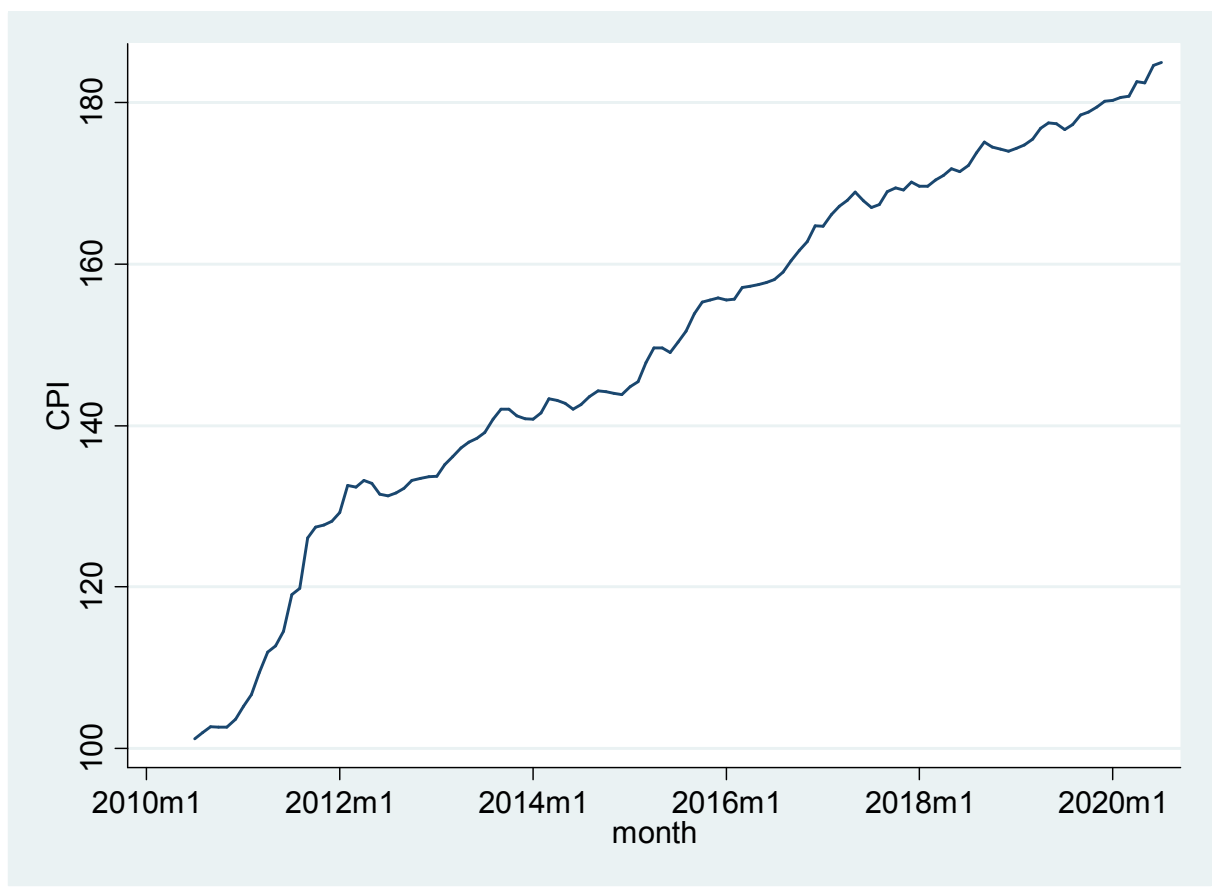

Figure 1. Uganda's Headline Consumer Price Index.

In Table 1, the computed Augmented Dickey-Fuller tstatistic for the headline CPI was-2.792 which was greater than the critical vales at $1 \%$ confirming that the series was not stationary. After first differencing the headline CPI becomes stationary since the computed Augmented DickeyFuller t-statistics was about- 8.34 which was less than the critical value at $1 \%$ of -3.50 .

Table 1. Augmented Dickey-Fuller t-statistics for headline CPI.

\begin{tabular}{|c|c|c|c|c|c|c|}
\hline & \multicolumn{3}{|l|}{ Values } & \multicolumn{3}{|c|}{ First Difference } \\
\hline & t-statistic & Test critical value $1 \%$ level & Prob* & t-statistic & Test critical value $1 \%$ level & Prob* \\
\hline Headline CPI & -2.792 & -3.503 & 0.0594 & -8.338 & -3.504 & 0.0000 \\
\hline
\end{tabular}

\subsection{Model Identification}

In addition to the trend observed above in chart 1 , the wavelike pattern of the first differenced series suggested presence of seasonality in the headline CPI series. Hence the Headline CPI was first differenced to control for trend followed by seasonal differencing (12 months) to control for seasonality before generation of the ACFs and PACFs as 
shown in Figures 2 and 3 respectively. Careful inspection of the ACFs revealed significant spikes at lag one, two and twelve months similarly the PACFs exhibited significant spikes at lags one, two and twelve months.

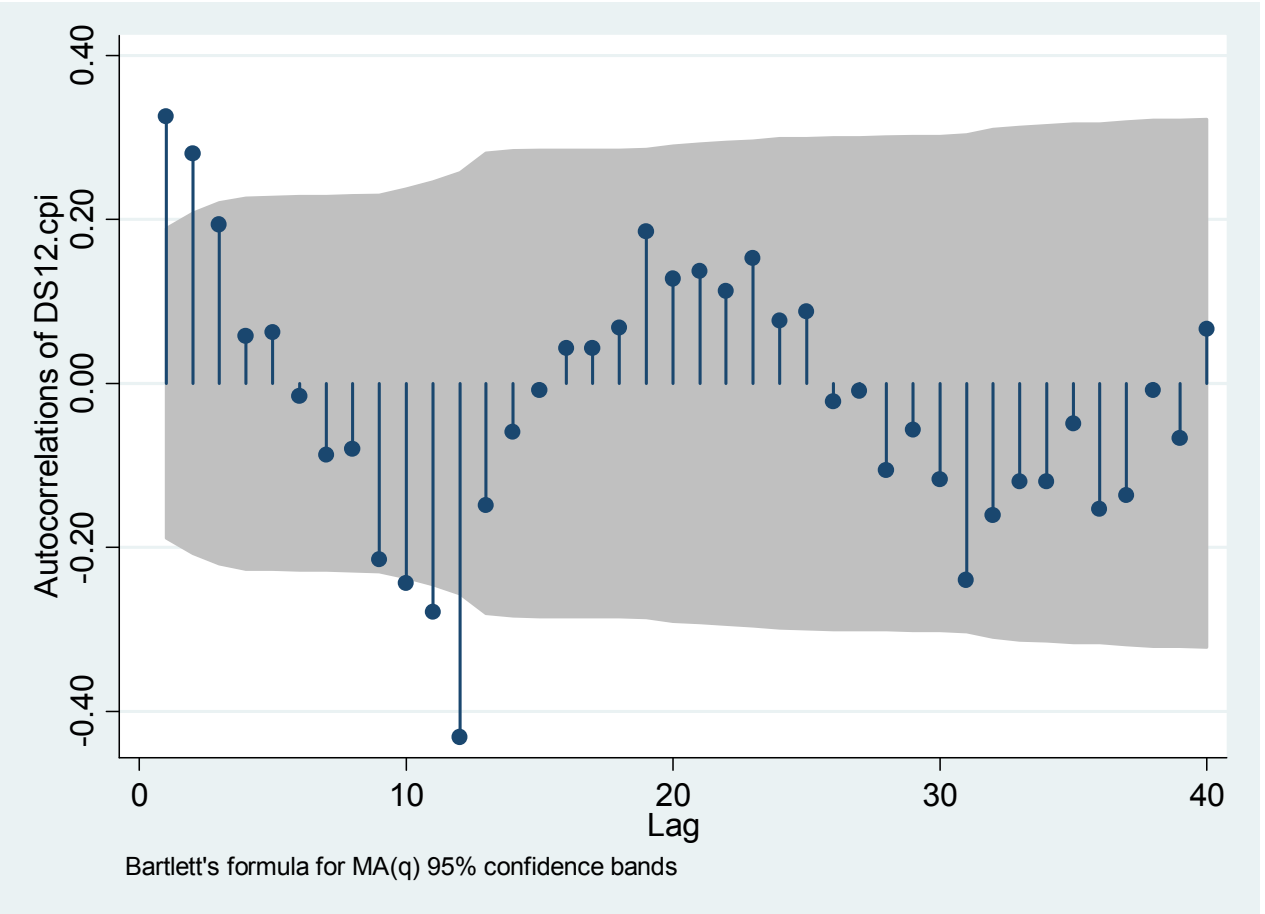

Figure 2. ACFs of first differenced and seasonally differenced Headline CPI series.

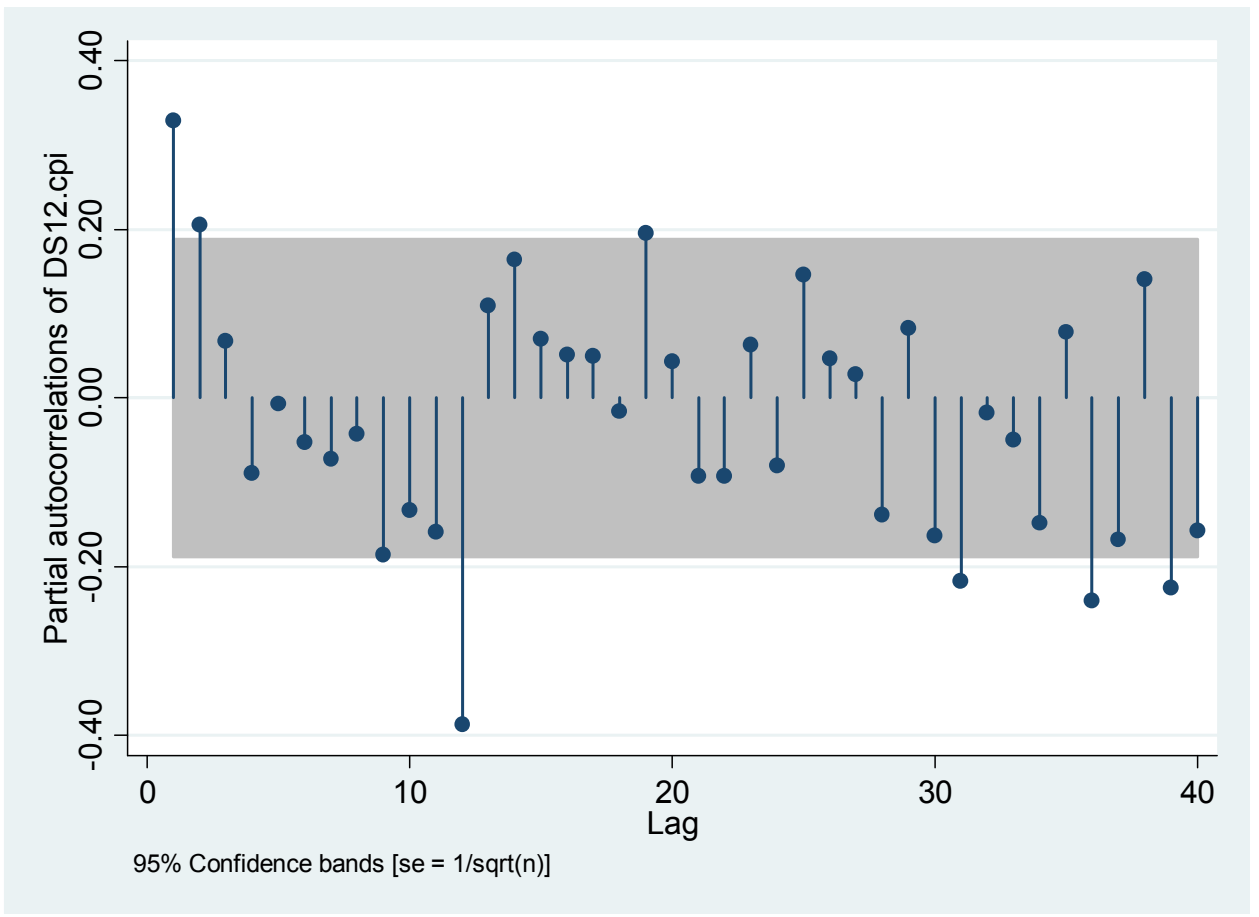

Figure 3. PACFs of first differenced and seasonally differenced Headline CPI series.

\subsection{Estimation}

The ACFs and PACFs showed that the model may not be purely AR or MA but mixed ARMA with seasonal factors. Consequently, a number of models were tested so as to identify the most suitable for the CPI series. Table 2 presents the results of different ARIMA models with the associated statistics. Seasonal ARIMA $(1,1,1) \times(0,1,1)_{12}$ with no constant was selected because it had the least AIC and BIC. Additionally, all the coefficients of the ARs and MAs were significant at $1 \%$ level. Furthermore, the plot of residuals in 
Figure 4 appeared normally distributed which was confirmed by the Bartlett's test for white noise at $1 \%$ level.

Table 2. The Seasonal ARIMA models (with their statistics) for Headline CPI series.

\begin{tabular}{|c|c|c|c|c|c|}
\hline \multicolumn{6}{|c|}{ Seasonal ARIMA Models } \\
\hline \multicolumn{2}{|c|}{ Characteristics } & $\operatorname{ARIMA}(1,1,1) \times(1,1,1)_{12}$ & $\operatorname{ARIMA}(1,1,0) \times(0,1,1)_{12}$ & $\operatorname{ARIMA}(1,1,1) \times(0,1,1)_{12}$ & $\begin{array}{l}\text { ARIMA }(1,1,1) \times(0,1,1)_{12} \\
\text { No constant }\end{array}$ \\
\hline \multirow{4}{*}{ AR (1) } & $\alpha_{1}$ & 0.0905 & 0.21658 & 0.78977 & 0.82128 \\
\hline & SE & 0.20773 & 0.09788 & 0.20719 & 0.16944 \\
\hline & t-value & 3.89 & 2.21 & 3.81 & 4.85 \\
\hline & $\mathrm{p}$ value & 0.000 & 0.027 & 0.000 & 0.000 \\
\hline \multirow{4}{*}{ MA (1) } & $\beta_{1}$ & -0.60649 & & 0.57265 & -0.59233 \\
\hline & SE & 0.25577 & & 0.26108 & 0.22149 \\
\hline & $\mathrm{t}$-value & -2.37 & & -2.19 & -2.67 \\
\hline & $\mathrm{p}$ value & 0.018 & & 0.028 & 0.007 \\
\hline \multirow{3}{*}{ SAR (12) } & $\alpha_{12}$ & -0.16618 & & & \\
\hline & $\begin{array}{l}\text { SE } \\
\text { t-value }\end{array}$ & $\begin{array}{l}0.12908 \\
-1.29\end{array}$ & & & \\
\hline & $\mathrm{p}$ value & 0.198 & & & \\
\hline \multirow{4}{*}{ SMA (12) } & $\beta_{12}$ & -0.723677 & -0.89371 & -0.84150 & -0.82409 \\
\hline & SE & 0.19131 & 0.29154 & 0.19608 & 0.17576 \\
\hline & $t$-value & -3.78 & -3.07 & -4.29 & $-4,69$ \\
\hline & $\mathrm{p}$ value & 0.000 & 0.002 & 0.000 & 0.000 \\
\hline \multirow{7}{*}{ Constant } & $\alpha_{0}$ & -0.05687 & -0.06961 & -0.05489 & \\
\hline & SE & 0.077999 & 0.04728 & 0.07419 & \\
\hline & $\mathrm{t}$-value & -0.73 & 11.47 & -0.74 & \\
\hline & $\mathrm{p}$ value & 0.466 & 0.141 & 0.459 & \\
\hline & AIC & 318.86 & 322.37 & 317.91 & 316.58 \\
\hline & $\mathrm{SBC}$ & 334.95 & 333.10 & 331.32 & 327.31 \\
\hline & Log Likelihood & -153.43 & -157.19 & -152.95 & -154.29 \\
\hline
\end{tabular}

Note: ARIMA - Autoregressive Integrated Moving Average, AIC-Akaike Information Criteria, SBC-Schwarz Bayesian Information Criterion and SE Standard Error

The selected Seasonal ARIMA model was as follows:

$$
\mathrm{y}_{\mathrm{t}}=0.82128 \mathrm{y}_{\mathrm{t}-1}+-0.59233 \varepsilon_{\mathrm{t}-1}+-0.82409 \varepsilon_{\mathrm{t}-13}
$$

where: y_Consumer Price Indices, $\varepsilon$ - Residuals, $\mathrm{t}$ - time and t-1-lagged time by one period

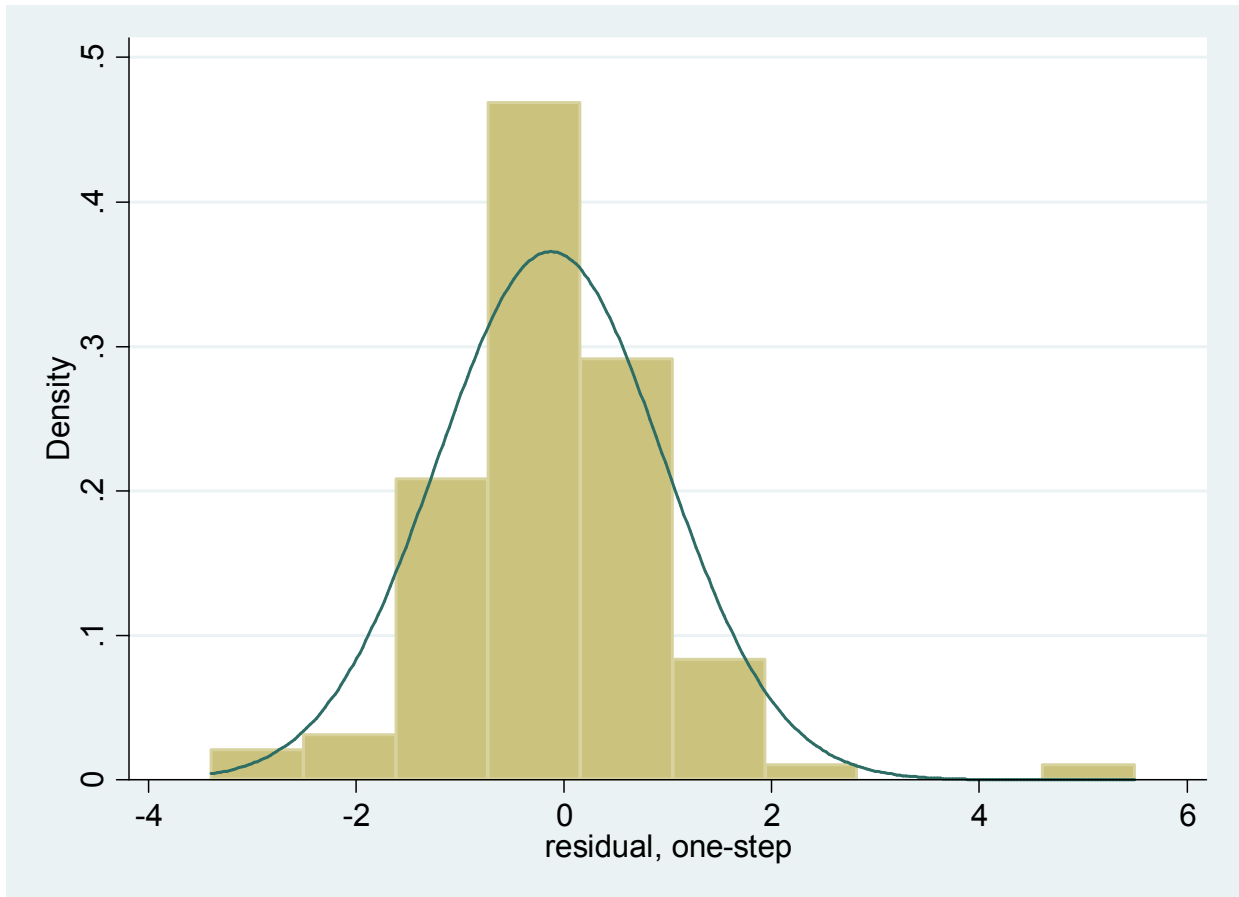

Figure 4. Residuals for the Seasonal ARIMA model. 


\subsection{Forecasts}

The Seasonal ARIMA $(1,1,1) \times(0,1,1)_{12}$ with no constant was selected for modeling the headline CPI. This model was used to make 12 months forecasts of the CPI. In Table 3, annual inflation was projected to fluctuate from 4.7 to 6 percent, which was close to the Central Bank's target of $5 \%$ in the medium term [3]. The fluctuations of the CPI are partly attributed to the seasonal nature of the food crops and related items which constitute about $10 \%$ of the weight of the headline CPI basket. Food crop production and supplies tend to oscillate along the seasonal patterns experienced in the country. The other major contributor to the seasonality is the exchange rate fluctuation which basically reflects the demand and supply of foreign currency.

Table 3. CPI and Inflation Forecasts.

\begin{tabular}{lll}
\hline Month & Headline CPI & Headline Inflation \\
\hline August 2020 & 186.122 & $5.0 \%$ \\
September 2020 & 187.924 & $5.3 \%$ \\
October 2020 & 188.528 & $5.4 \%$ \\
November 2020 & 188.790 & $5.2 \%$ \\
December 2020 & 189.410 & $5.1 \%$ \\
January2021 & 189.723 & $5.2 \%$ \\
February 2021 & 190.560 & $5.5 \%$ \\
March 2021 & 191.651 & $6.0 \%$ \\
April 2021 & 192.793 & $5.6 \%$ \\
May 2021 & 193.176 & $5.9 \%$ \\
June 2021 & 193.406 & $4.7 \%$ \\
July 2021 & 193.849 & $4.8 \%$ \\
\hline
\end{tabular}

\section{Discussions}

The results of the study were consistent with the CPI models estimated by other authors for Nigeria, Rwanda and Somaliland $[12,14,16]$. However, those models did not have a seasonal component in the ARIMA models. The study for Nigeria found that inflation increased modestly all through the period $1990-2010$ [11]. Inflation forecasts for 2020/21 for Uganda averaged $5.3 \%$ which is more than twice $(2.4 \%)$ the average forecasts by Adams et. al. for Nigeria for $2011-$ 2013 [11]. Seasonality was found to be significant in the ARIMA model for Uganda's CPI and the forecasts should incorporate it for better predictions.

\section{Conclusion}

The central bank in Uganda makes monthly monetary policy stance pronouncements which are anchored on inflation rates. Therefore, accurate forecasts in the medium term are very important for this purpose and for making decisions by other users in the economy. ARIMA models are univariate and they rely on the time series mostly to come up with estimates of coefficients and forecasts. The Seasonal ARIMA $(1,1,1) \times(0,1,1)_{12}$ was found to be the most appropriate model hence it was used to forecast CPI and hence inflation. We recommend this model to Uganda Bureau of Statistics and Central Bank to use it to make forecasts and disseminate to the users. In conclusion, generally good forecasts are vital for better resource allocation, planning and decision making.

\section{References}

[1] Government of Uganda, "Investment Code Act 1991," Uganda Printing and Publishing Corporation, Kampala, 1991.

[2] Uganda Revenue Authority, "A Guide on Tax Incentives/Exemptions Avialbale to the Uganda Investors," 9 September 2019. [Online]. Available: https://www.ugandainvest.go.ug/wp-

content/uploads/2019/12/Tax-Incentives-for-2019.pdf.

[3] Bank of Uganda, "State of the Economy," Bank of Uganda, Kampala, 2019.

[4] A. Mugume, "The Inflation Targeting Monetary Policy Framework, Seminar for Business Editors and Reporters," Bank of Uganda, Kampala, 2011.

[5] D. N. Mubiru, E. Komutunga, A. Agona, A. Apok and T. Ngara, "Characterising agrometeorological climate risks and uncertainties: Crop production in Uganda," South African Journal of Science, pp. 1-11, 2011.

[6] Bank of Uganda, "monetary policy," 17 August 2020. [Online]. Available: https://www.bou.or.ug/bou/bouwebsite/MonetaryPolicy/.

[7] International Labour Organization, "Consumer Price Index Manual, Theory and Practice," International Labour Organization, Geneva, 2004.

[8] R. Adhikari and K. R. Agrawal, "An Introductory Study on Time Series Modeling and Forecasting," LAP LAMBERT Academic Publishing, New Dehli, 2013.

[9] E. Stellwagen and L. Tashman, "ARIMA: The Models of Box and Jenkins," The International Journal of Applied Forecasting, 2013.

[10] D. C. Montgomery, C. L. Jennings and M. Kulahci, Introduction to Time Series Analysis \& Foresating, Canada: John Wiley \& Sons, 2008.

[11] Uganda Bureau of Statistics, "Consumer Price Index: 2009/2010=100: July 2020," Uganda Bureau of Statitics, Kampala, 2020.

[12] N. Habimana, A. Wanjoya and A. Waititu, "Modeling and Forecasting Consumer Price Index (Case Study of Rwanda," American Journal of Theoretical and Applied Statistics, pp. 101-107, 2026.

[13] X. Zhang, "CPI Prediction Based on ARIMA Model," in 7th International Conference on Education, Management, Information and Computer Science (ICEMC 2017), Shanghai, 2017.

[14] S. O. Adams, A. Awujola and A. I. Alumgudu, "Modeling Nigeria's Consumer Price Index using ARIMA Model," International Journal of Development and Economic Sustainability, pp. 37-47, 2014.

[15] T. Nyoni, "ARIMA Modeling and Focasting of CPI in Germany," Munich Personal RePEc Archive, pp. 1-13, 2019. 
[16] J. Mohamed, "Time Series Modeling and Forecasting of Somaliland Consumer Price Index: A comparision of ARIMA and Regression with ARIMA Error," American Journal of Theoretical and Applied Statistics, pp. 143-153, 2020. 